\title{
Vertically polarized omni-directional loop slot array antenna for mobile base station
}

\author{
Ryosuke Kaneda ${ }^{\text {a) }}$ and Hiroyuki Arai \\ Graduate School of Engineering, Yokohama National University, \\ 79-5 Tokiwadai, Hodogaya-ku, Yokohama 240-8501, Japan \\ a)kaneda-ryosuke-wb@ynu.jp
}

Abstract: A slim vertically polarized omni-directional feed circuit integrated loop slot antenna for mobile base station is proposed in this paper. Picocell antenna requires a compact and slim structure, because its installation space is limited in urban areas. The proposed antenna consists of loop slots and patches to improve omni-directional pattern and bandwidth. In order to enhance the directivity gain, we arrange two loop slot antennas to be excited out of phase by a microstrip line. The size of the antenna is only $0.17 \lambda \times 1.45 \lambda$. This antenna realizes more than $15 \%$ relative bandwidth, and an omni-directional pattern in both simulation and measurement results.

Keywords: vertically polarized, omni-directional, loop slot, parasitic elements, feeding circuit integrated antenna

Classification: Antennas and Propagation

\section{References}

[1] H. Kakehuda, A. Shimamura, T. Tanaka, and Y. Ebine, "A design method of dual polarized omni-directional antenna for radio blind areas," Soc. Conf. IEICE., vol. B-1-79, 2009.

[2] M. Polivka and A. Holub, "Collinear and coparallel principles in antenna design,” PIERS Proc., pp. 337-341, 2007.

[3] Y. Yokoo and H. Arai, "Multi-frequency loop slot antenna with omnidirectional pattern," IEICE trans. Comm., vol. J88-B, no. 9, pp. 1718-1727, 2005.

[4] H. D. Chen, C. Y. D. Sim, J. Y. Wu, and T. W. Chiu, "Broadband high-gain microstrip array antennas for WiMAX base station," IEEE Trans. Antennas Propag., vol. 60, no. 8, pp. 3977-3980, 2012. DOI:10.1109/TAP.2012.2201116

[5] C.-F. Huang and W.-Y. Chen, "A wideband microstrip array based on air substrate for wireless base-station applications," IEEE EuCAP, pp. 1268-1271, 2013. 


\section{Introduction}

Picocell antennas require a slim structure and an omni-directional pattern in horizontal plane. A dipole and a collinear array antenna are generally used as vertically polarized omni-directional base station antennas [1,2].

A vertically polarized omni-directional loop slot antenna was proposed by combining the loop slot with parasitic patch and feed lines for multi-band application [3]. To realize a simple feeding circuit for omni-directional pattern antenna, we propose a feed circuit integrated antenna.

This paper presents a simple array feed network to enhance the directivity in vertical plane and a defected ground plane to suppress unwanted mode radiation in horizontal plane. The proposed antenna geometry is compared with a tournament feed network in simulation and measurement.

\section{Antenna design}

We propose out of phase excitation for vertically polarized omni-directional loop slot array antenna with parasitic elements. In most applications $[4,5]$, the tournament feeding circuit is generally used as feeding circuit of array antenna. Fig. 1(a) shows the two-loop slots array antenna using a tournament feeding circuit. One of the problems of this feeding structure is large antenna width because feeding circuit is arranged along the loop slot. The width of the antenna has an influence on radiation pattern in horizontal plane. To reduce the antenna width, we propose a new feeding circuit for loop slot antenna.

Fig. 1(b) shows the geometry of the proposed antenna which has a slim structure $(0.17 \lambda \times 1.45 \lambda)$.

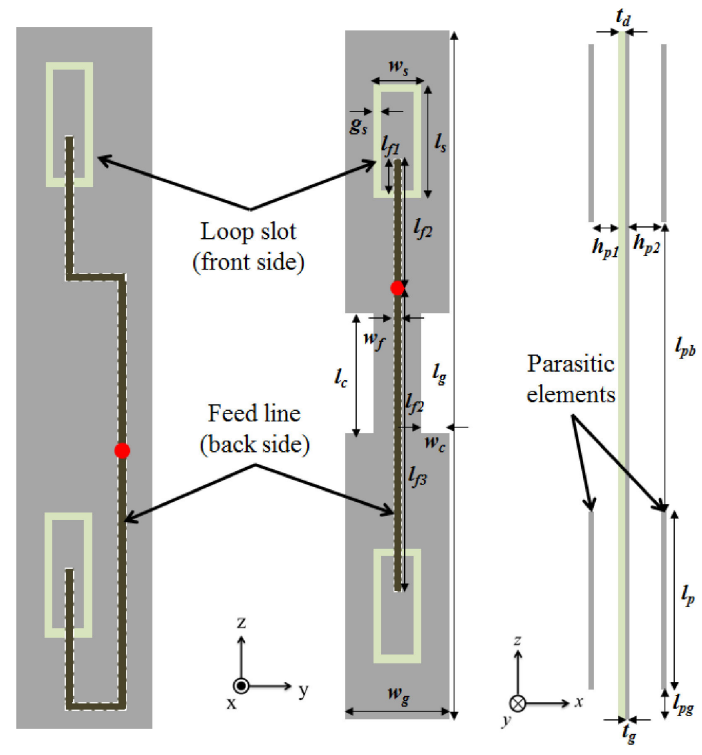

(a) Tournament feeding antenna

(b) Proposed feeding antenna

Fig. 1. Antenna configuration.

$l_{g}=1.45 \lambda, w_{g}=0.17 \lambda, l_{s}=0.27 \lambda, w_{s}=0.08 \lambda, g_{s}=0.01 \lambda$,

$l_{f 1}=0.08 \lambda, l_{f 2}=0.29 \lambda, l_{f 3}=0.61 \lambda, w_{f}=0.01 \lambda, l_{c}=0.13 \lambda$,

$w_{c}=0.06 \lambda, \quad l_{p b}=0.50 \lambda, \quad l_{p g}=0.11 \lambda, \quad l_{p d}=0.36 \lambda, \quad h_{p 1}=$ $0.06 \lambda, h_{p 2}=0.05 \lambda, t_{g}=0.5 \mathrm{~mm}, t_{d}=0.8 \mathrm{~mm}$ 
This antenna has two loop slots excited by a microstrip line on the back side of a dielectric substrate with relative dielectric constant of $\varepsilon r=2.6$. Each slot is located at a distance of $0.9 \lambda$ from each other. Two elements are excited out of phase to excite both antenna elements by a straight microstrip line as shown in Fig. 1(b). The parasitic elements are placed at both sides of the substrate. The parasitic elements are excited by the field of the loop slots. At the center of the structure of the proposed antenna, two rectangle parts (length $=l_{c}$, width $=w_{c}$ ) are removed to reduce the unwanted mode radiation. The current distributions are shown in Fig. 2(a), which shows that the proposed structure helps in removing standing wave on the ground plane. Fig. 2(b) shows that radiation pattern is improved by proposed structure.

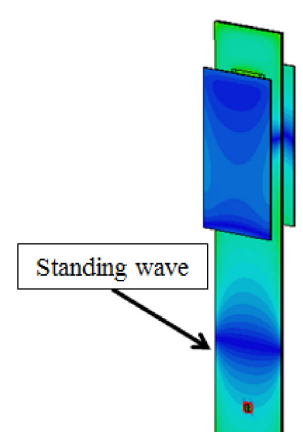

(i) Original structure

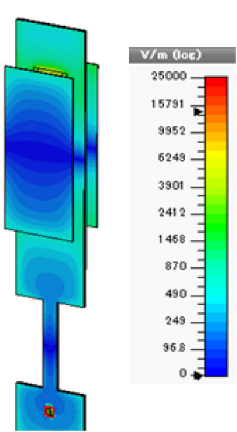

(ii) Proposed structure

(a) The current distribution $\left(0.93 \mathrm{f}_{0}\right)$

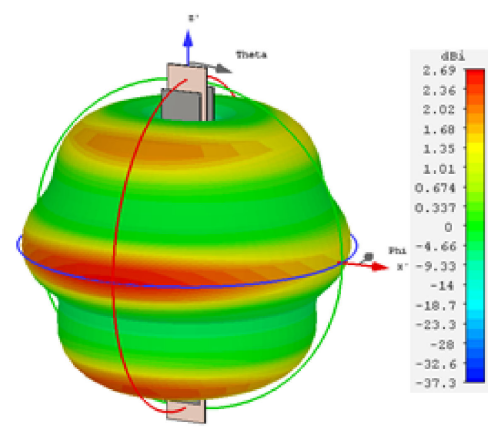

(i) Original structure

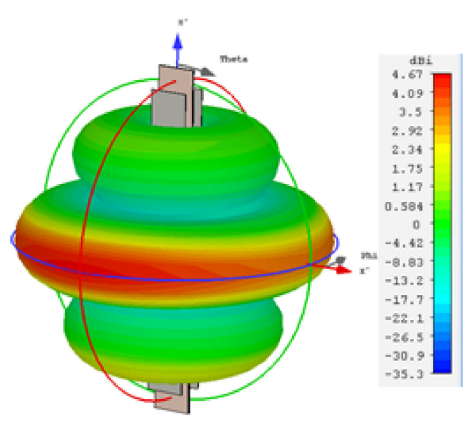

(ii) Proposed structure

(b) The radiation pattern $\left(0.93 \mathrm{f}_{0}\right)$

Fig. 2. The current distribution and radiation pattern.

\section{Simulation and experiment results}

In this section, we compare the proposed feeding antenna with a tournament feeding antenna in simulation and measurement. Fig. 3(a) shows the reflection characteristics. The bandwidth of the proposed antenna is wider than tournament one due to its simple feeding circuit. The relative bandwidth of the proposed antenna is more than $15 \%$ for the reflection below $-10 \mathrm{~dB}$ in both simulation and measurement. The reflection characteristic shows two resonant frequencies. The first one is excited by parasitic elements $(x>0)$ coupled with the loop slot, and the other is given by the coupling between the parasitic elements $(\mathrm{x}<0)$ on feeding side and the feeding line. 
The radiation patterns in horizontal plane are shown in Fig. 3(b) for two frequencies at $0.93 \mathrm{f}_{0}$ and $1.10 \mathrm{f}_{0}$. It shows that the proposed antenna provides omni-directional pattern better than tournament one because the resonance of the ground plane is suppressed by the proposed geometry. The simulation results are similar with measurement in reflection characteristics. The radiation from the parasitic element on the feeding side is stronger than the side one at high frequency, thus the parasitic element positions are adjusted as $h_{p 1}=0.06 \lambda$ and $h_{p 2}=0.05 \lambda$ to reduce the pattern deviation in horizontal plane. The radiation patterns in vertical plane are shown in Fig. 3(c). In lower frequency, side lobe of proposed antenna is larger than tournament one due to the radiation by the feeding circuit.

The gain deviations of the proposed antenna in both simulation and measurement in horizontal plane are less than $3 \mathrm{~dB}$ for two frequencies. The gain deviations

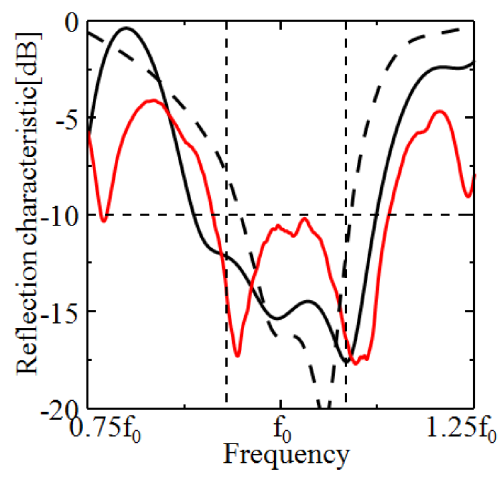

(a) Reflection characteristic.
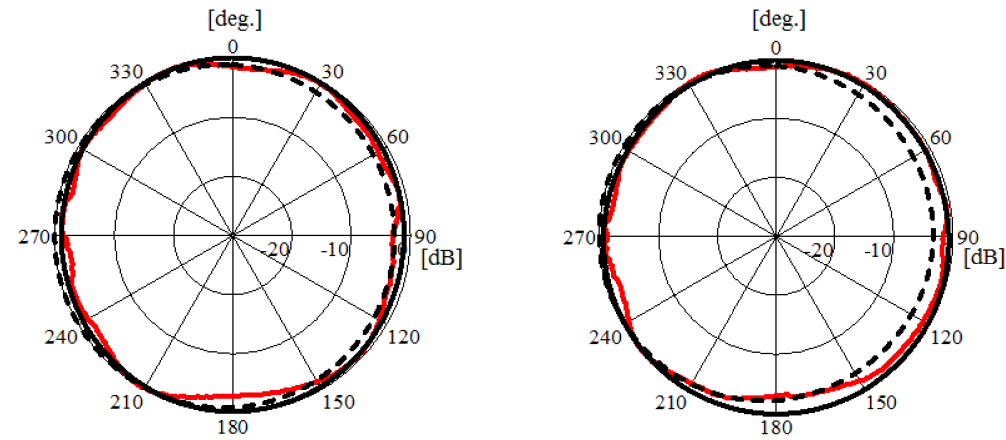

(b) Radiation pattern in horizontal plane (xy-plane).
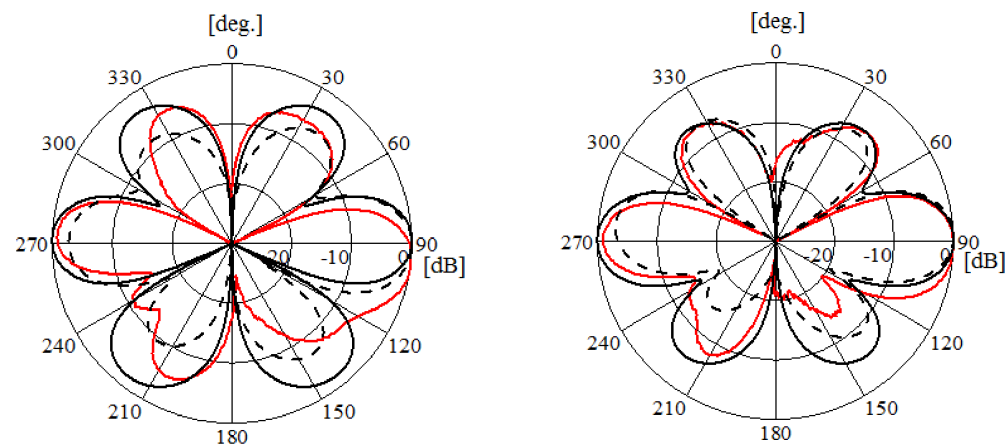

(c) Radiation pattern in horizontal plane (yz-plane). 
for the simulation in horizontal plane are smaller than the measurement due to the influence of coaxial cable in experimental system.

\section{Conclusion}

We proposed a slim vertically polarized omni-directional feed circuit integrated array antenna for mobile base station. This antenna had two loop slots and patches excited by a microstrip line on the back side of dielectric substrate. The two elements were excited out of phase by the straight microstrip line. This antenna provided more than $15 \%$ relative bandwidth, and an omni-directional pattern in both simulation and measurement results. 